\title{
Padrões de distribuição geográfica das espécies de Euploca e HeLIOTRopium (HELIOTROPIACEAE) No BRASIL
}

\author{
José Iranildo Miranda de Melo ${ }^{1}$, Marccus Alves ${ }^{2}$ \& João Semir ${ }^{3}$
}

\begin{abstract}
Resumo
(Padrões de distribuição geográfica das espécies de Euploca e Heliotropium (Heliotropiaceae) no Brasil) Baseando-se na análise de espécimes advindos de 71 herbários nacionais e estrangeiros, trabalhos de campo e literatura especializada, foram determinados os padrões de distribuição geográfica das espécies dos gêneros Euploca e Heliotropium no Brasil. Foram detectados quatro padrões de distribuição geográfica e onze padrões biogeográficos. O centro de diversidade das espécies de Euploca no Brasil é a Região Nordeste. As espécies do gênero Heliotropium concentram-se, na sua maioria, na Região Sul, com o estado do Rio Grande do Sul representando o centro de diversidade do gênero no país. São apresentadas tabelas, mapas e discussões sobre a distribuição e respectivos padrões biogeográficos reconhecidos.
\end{abstract}

Palavras-chave: Angiospermas, neotrópicos, padrões biogeográficos.

\section{Abstract}

(Geographic distribution patterns of species of Euploca and Heliotropium (Heliotropiaceae) in Brazil) Based on an analysis of specimens from seventy-one national and international herbaria, field work and specialized literature, geographic distribution patterns of the species of the genera Euploca and Heliotropium in Brazil were determinate. Four geographic patterns and eleven biogeographic patterns were detected. The center of diversity of Euploca, in Brazil, is in the Northeast region. Species of the genus Heliotropium occur generally in the South region, with the center of diversity of the genus being found in Rio Grande do Sul State. Tables, maps and a discussion about the respective distribution and biogeographic patterns uncovered in this study are presented.

Key words: flowering plants, neotropics, biogeography patterns.

\section{INTRODUÇÃO}

A família Heliotropiaceae com cinco gêneros e cerca de 450 espécies se encontra distribuída nas zonas tropical, subtropical e temperada, e ocorre, geralmente, em ambientes xéricos (Miller 2003). No Brasil, está representada pelos gêneros Euploca Nutt. (17 spp.), Heliotropium L. (9), Tournefortia L. (5) e Myriopus Small (20), sendo este último sugerido, mas ainda não revalidado, nos estudos filogenéticos realizados por Hilger \& Diane (2003).

Segundo Sylvestre (2002) a análise dos padrões de distribuição geográfica surge comouma importante ferramenta na definição dos graus de endemismo, inferindo diretamente na indicação de áreas prioritárias para conservação. Nesse sentido, Thomas \& Magill (2002) observam que as pesquisas em taxonomia vegetal são as que possibilitam enumerare distinguir espécies, mapear a distribuição e necessidades de habitats e ainda documentar os usos e raridade - todos dados críticos para estabelecer prioridades de conservação plausíveis. Nesse sentido, a determinação dos padrões de distribuição geográfica de diferentes grupos taxonômicos configura-se de expressiva importância. Isso pode gerar subsídios que visem à implantação de Unidades de Conservação (UC), como também à realização de inventários florísticos e estudos sobre dinâmica de populações e sistemas reprodutivos.

Artigo recebido em 01/2009. Aceito para publicação em 11/2009.

${ }^{1}$ Parte da tese de Doutorado do autor, Programa de Pós-Graduação em Botânica da Universidade Federal Rural de Pernambuco/Universidade Estadual da Paraíba, Centro de Ciências Biológicas e da Saúde, Depto. Biologia, Av. das Baraúnas 351, Campus Universitário, Bodocongó, 58109-753, Campina Grande, PB, Brasil. Autor para correspondência: iranildo_melo@hotmail.com

${ }^{2}$ Universidade Federal de Pernambuco, Depto. Botânica, Av. Prof. Moraes Rego s/n, Cidade Universitária, 50670-901, Recife, PE, Brasil.

${ }^{3}$ Universidade Estadual de Campinas, Depto. Botânica, Instituto de Biologia, Programa de Pós-Graduação em Biologia Vegetal, C.P. 6109, 13083-970, Campinas, SP, Brasil. 
O Brasil tem a flora mais rica do mundo e estimativas atuais indicam a existência de 55.000-60.000 espécies de angiospermas (Giulietti et al. 2005). Apesar disto, no Brasil, os estudos sobre padrões de distribuição geográfica são ainda escassos, dos quais se destacam: Acevedo-Rodríguez (1990), Barros (1990), Boechat \& Longhi-Wagner (2000), Flores \& Miotto (2005), Giulietti \& Pirani (1988), Longhi-Wagner \& Zanin (1998), Longhi-Wagner (1990), Marchioretto et al. (2004), Pirani (1990), Sakuragui (2001) e Wanderley (1990).

Este trabalho objetivou reconhecer os padrões de distribuição geográfica das espécies dos gêneros Euploca e Heliotropium no Brasil e, desse modo, contribuir para o conhecimento da biogeografia de Heliotropiaceae.

\section{Material e Métodos}

A distribuição geográfica das espécies estudadas foi baseada principalmente em coleções depositadas nos principais herbários brasileiros e do exterior, num total de 64 instituições mencionadas a seguir (segundo Holmgren et al. 2006): ALCB, ASE, BAB, BHCB, CEN, CEPEC, CESJ, COR, CPAP, CTES, EAC, EAN, ESA, ESAL, GH, GUA, GUYN, HAS, HBR, HRB, HRCB, HTO, HUEFS, HUFU, IAC, IAN, IBGE, ICN, INPA, IPA, JPB, K, MAC, MBM, MBML, MG, MO, MOSS, MY, NY, PACA, PEL, PEUFR, PORT, R, RB, RFA, SJRP, SMDB, SP, SPF, SPSF, TEPB, UB, UCS, UEC, UFP, UFRN, US, VEN, VIC, VIES, Z. Além destes, foram consultados sete herbários nacionais não listados em Holmgren et al. (2006): CH, CNPF, HBH, HFC, HST, MHN, MUFAL e UFMS.

A análise da distribuição geográfica foi complementada por informações contidas na literatura especializada (Johnston 1928; Melo \& Sales 2005; Melo \& Andrade 2007), além de espécimes obtidos durante excursões de coleta realizadas em diferentes estados do nordeste do Brasil (Ceará, Rio Grande do Norte, Paraíba, Pernambuco, Alagoas, Bahia). Os padrões biogeográficos seguem os estabelecidos por Cabrera \& Willink (1980) e Morrone (1999), e estão determinados com base exclusivamente em espécimes examinados.

As coordenadas geográficas foram obtidas a partir dos rótulos das exsicatas ou através do sítio http://paginas.terra.com.br/ educação/Astronomia/latUF.html. A plotagem das espécies foi feita com base no mapa da América do Sul (modificado da Flora Neotropica).

\section{Resultados e Discussão}

Os gêneros Euploca e Heliotropium são encontrados por todas as Regiões e estados do país (Tab. 1). Estão representados nos domínios: Amazônico e Chaquenho, englobando quatro das nove províncias biogeográficas (Amazônica, Cerrado, Atlântica, Paranaense) propostas para o domínio Amazônico e apenas uma província (Caatinga) para o domínio Chaquenho (Cabrera \& Willink 1980).

Euploca e Heliotropium apresentam no Brasil quatro padrões de distribuição geográfica e onze padrões biogeográficos (Figs. 1 e 2, Tab. 2): contínuo e amplo (Amazônico-Cerrado-CaatingaAtlântico-Paranaense-Pampeano, AmazônicoCerrado-Atlântico-Paranaense, AmazônicoAtlântico-Caatinga, Atlântico-Caatinga-Paranaense, Cerrado-Caatinga-Atlântico-ParanaensePampeano, Cerrado-Caatinga-Paranaense), contínuo e restrito (Cerrado-Caatinga, PampeanoParanaense, Pampeano), contínuo e muito restrito (endemismos local e regional), além de um táxon geograficamente disjunto.

Heliotropium curassavicum L. distribui-se nas porções tropicais e subtropicais da América, África e Ásia, associada geralmente a ambientes salinos. No Brasil, ocorre na Região Nordeste em áreas de Caatinga, aliada especialmente a habitats salinos em locais em processo de desertificação, e nos Campos Sulinos. Esta espécie é a única neste estudo que compreende o padrão de distribuição geográfica disjunto (Fig. 1, Tab. 2).

Heliotropium indicum L. possui distribuição cosmopolita, associada às regiões tropicais, enquanto que Euploca filiformis (Lehm.) J.I.M. Melo \& Semir ocorre desde o México, Antilhas, até Argentina (M.W. Frohlich com. pess.) caracterizando uma distribuição interamericana. No Brasil, ambas as espécies 


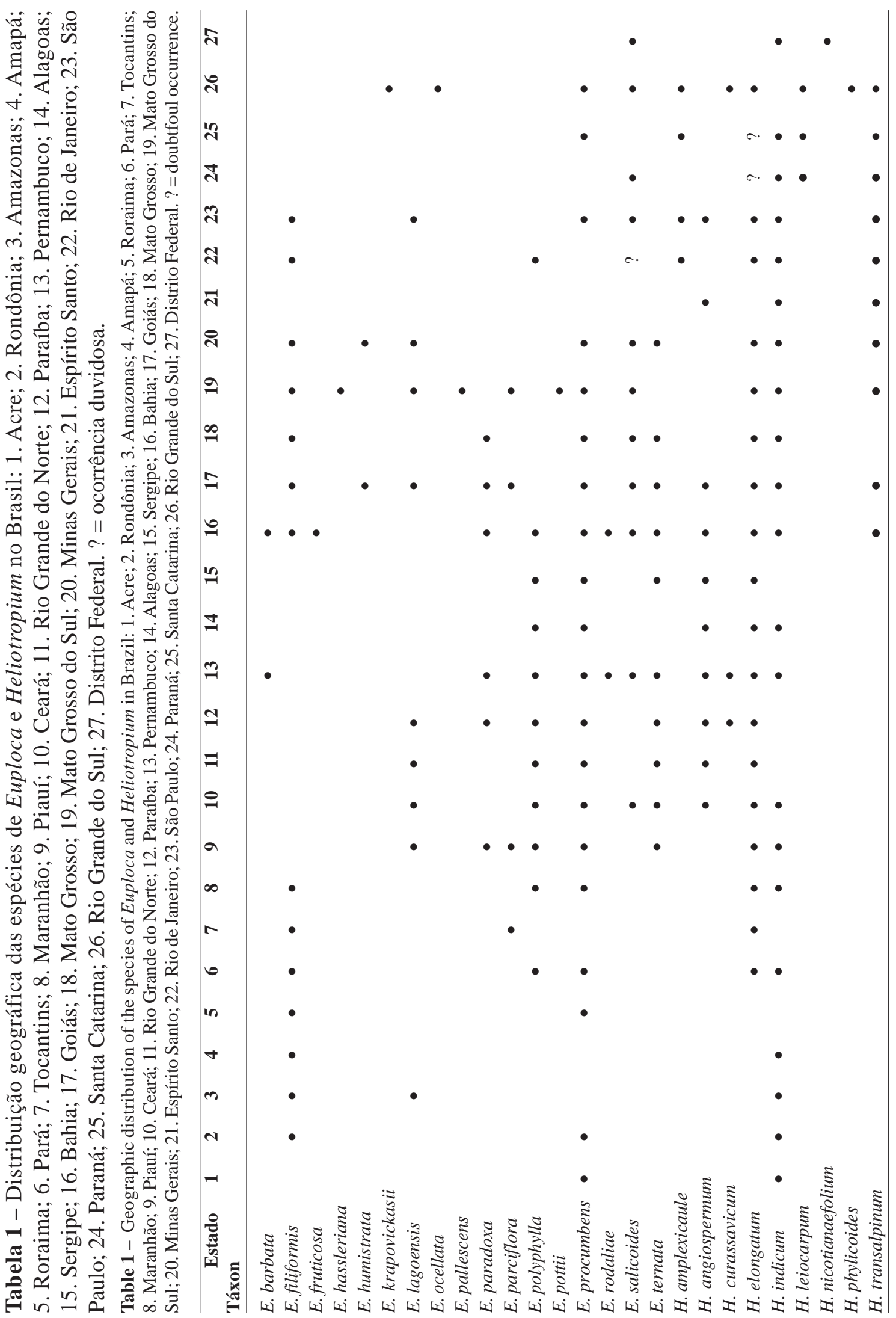




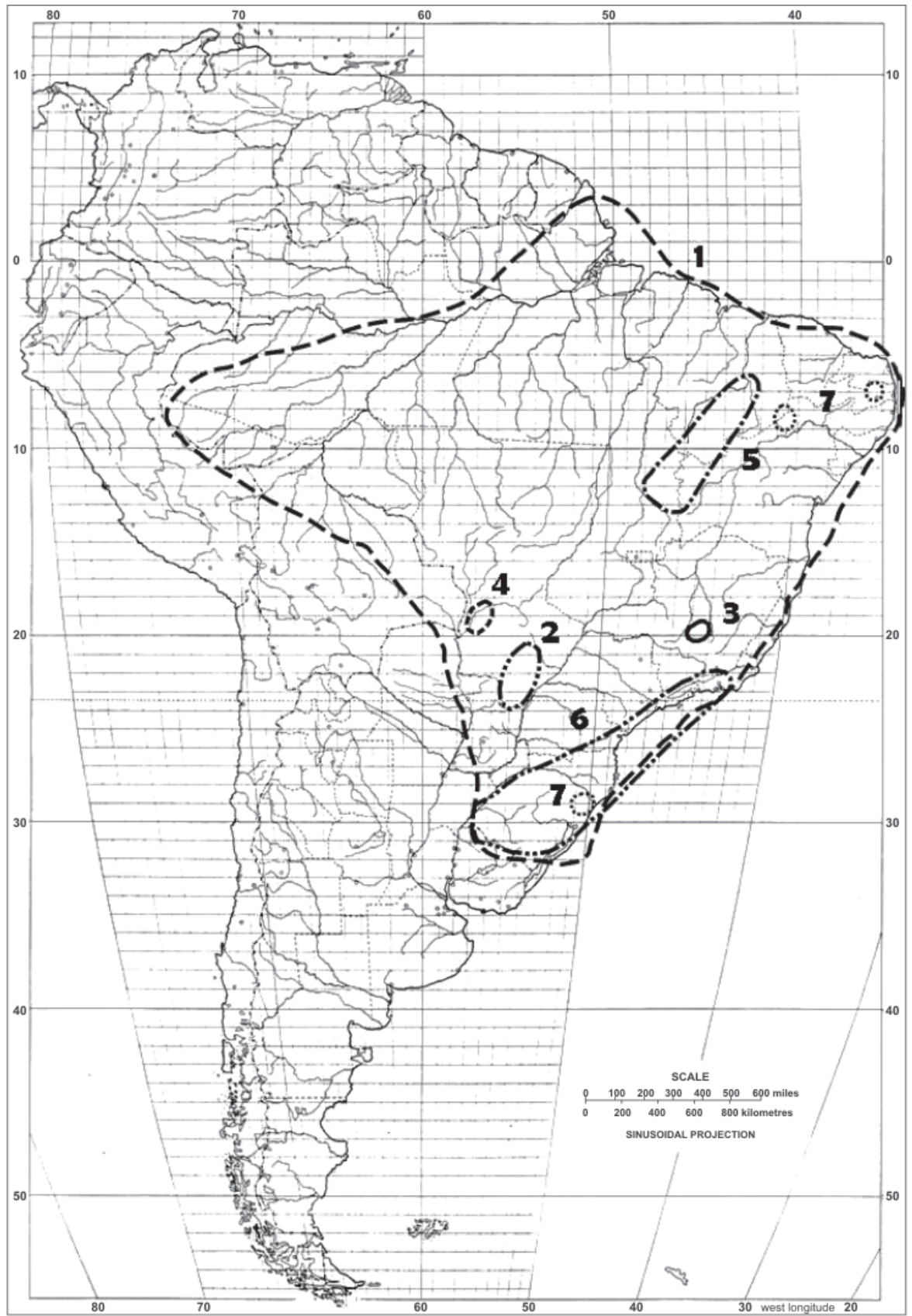

Figura 1 - Padrões de distribuição geográfica das espécies de Euploca e Heliotropium no Brasil: (1) E. filiformis (Lehm.) J.I.M. Melo \& Semir, E. procumbens (Mill.) Diane \& Hilger, Heliotropium elongatum (Lehm.) I.M. Johnst. e H. indicum L.: _ _ _ _ ; (2) E. hassleriana (Chodat) J.I.M. Melo \& Semir: _... _ . . _ ; (3) E. humistrata (Cham.) J.I.M. Melo \& Semir:;

(4) E. pallescens (I.M. Johnst.) J.I.M. Melo \& Semir e E. pottii J.I.M. Melo \& Semir:- - - - _ _; (5) E. parciflora (Mart.) J.I.M. Melo \& Semir: _._._._; (6) H. amplexicaule Vahl: _.. _ . _ ...; (7) H. curassavicum L.: ...........

Figure 1 - Patterns of geographic distribution of the species of Euploca and Heliotropium in Brazil: (1) E. filiformis (Lehm.) J.I.M. Melo \& Semir, E. procumbens (Mill.) Diane \& Hilger, Heliotropium elongatum (Lehm.) I.M. Johnst. e H. indicum L.: _-_-_; (2) E. hassleriana (Chodat) J.I.M. Melo \& Semir: _...-...-; (3) E. humistrata (Cham.) J.I.M. Melo \& Semir: _ ; (4) E. pallescens (I.M. Johnst.) J.I.M. Melo \& Semir e E. pottii J.I.M. Melo \& Semir: - - _-_._. . ; (5) E. parciflora (Mart.) J.I.M. Melo \& Semir: _._._._; (6) H. amplexicaule Vahl: _... _ _ ; (7) H. curassavicum L.: ............ 


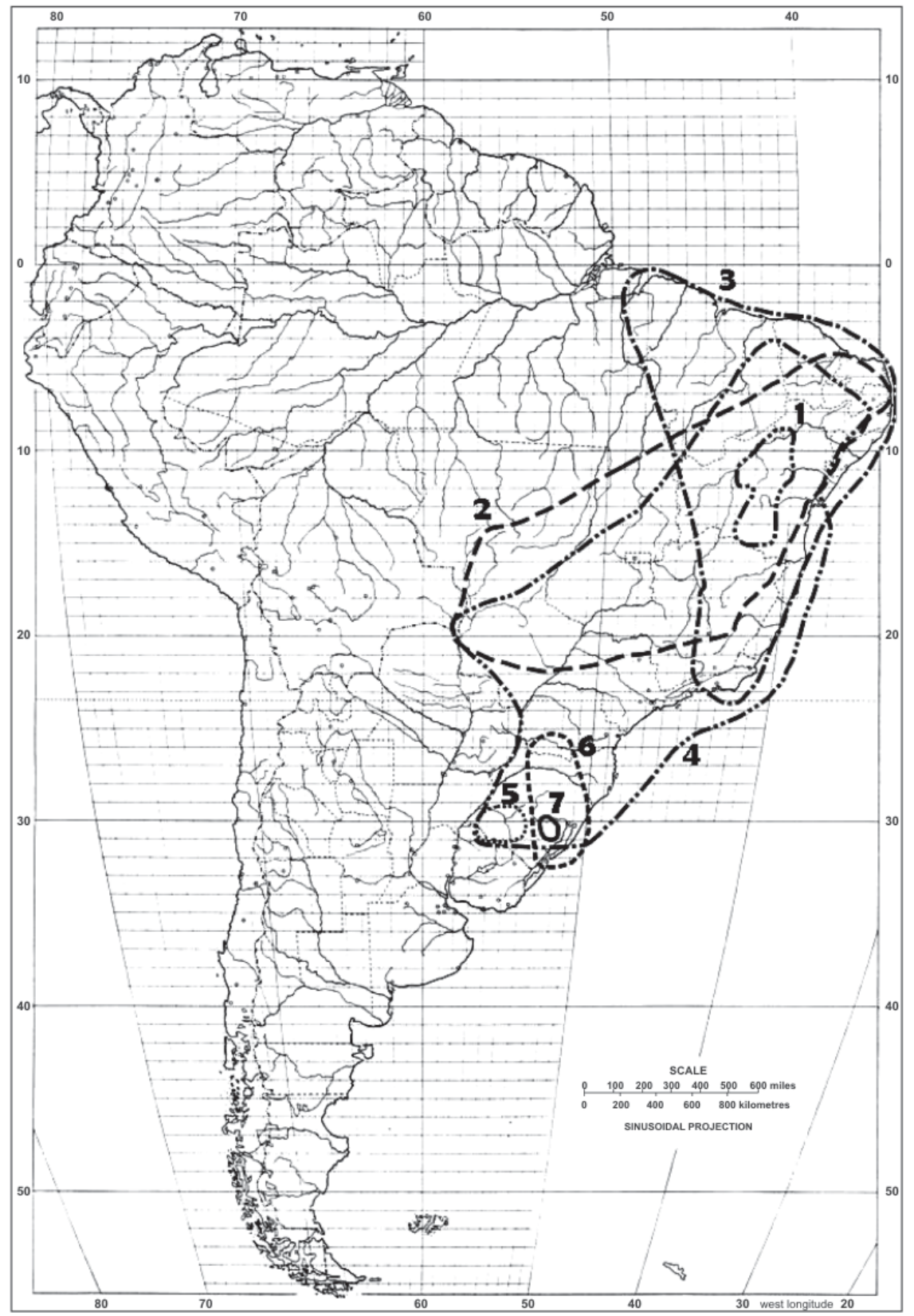

Figura 2 - Padrões de distribuição geográfica das espécies de Euploca e Heliotropium no Brasil: (1) E. barbata (DC.) J.I.M. Melo \& Semir e E. fruticosa (L.) J.I.M. Melo \& Semir: _... _... _; (2) E. lagoensis (Warm.) Diane \& Hilger, E. salicoides (Cham.) J.I.M. Melo \& Semir e H. transalpinum Vell.: _ - _ _ - ; (3) E. polyphylla (Lehm.) J.I.M. Melo \& Semir e H. angiospermum Murray: _. _. _. _ ; (4) E. paradoxa (Mart.) J.I.M. Melo \& Semir, E. rodaliae J.I.M. Melo \& Semir e E. ternata (Vahl) J.I.M. Melo \& Semir: _.. _ . _ . . ; (5) E. krapovickasii J.I.M. Melo \& Semir, E. ocellata (Cham.) J.I.M. Melo \& Semir e H. phylicoides Cham.: ; (6) H. leiocarpum Morong: ; (7) $H$. nicotianaefolium Poir.:

Figure 2 - Patterns of geographic distribution of the species of Euploca and Heliotropium in Brazil: (1) E. barbata (DC.) J.I.M. Melo \& Semir e E. fruticosa (L.) J.I.M. Melo \& Semir:_..._.... ; (2) E. lagoensis (Warm.) Diane \& Hilger, E. salicoides (Cham.) J.I.M. Melo \& Semir e H. transalpinum Vell.: _ - - _ - ; (3) E. polyphylla (Lehm.) J.I.M. Melo \& Semir e H. angiospermum Murray: _._._._; (4) E. paradoxa (Mart.) J.I.M. Melo \& Semir, E. rodaliae J.I.M. Melo \& Semir e E. ternata (Vahl) J.I.M. Melo \& Semir: _....... ; (5) E. krapovickasii J.I.M. Melo \& Semir, E. ocellata (Cham.) J.I.M. Melo \& Semir e H. phylicoides Cham.: 
apresentam ampla e contínua distribuição em diversas formações vegetais. De acordo com a classificação proposta por Cabrera \& Willink (1980) e Morrone (1999), enquadram-se no padrãoAmazônico-Cerrado-Atlântico-Paranaense (Fig. 1, Tab. 2), com distribuição geográfica ampla e contínua.

Euploca fruticosa (L.) J.I.M. Melo \& Semir ocorre desde o sul dos Estados Unidos até a América do Sul (Venezuela e Brasil), inclusive nas Antilhas. No Brasil, no entanto, é encontrada apenas no sudoeste baiano, na vegetação de Caatinga e enquadra-se no padrão biogeográfico caatinga com distribuição geográfica restrita (Fig. 2, Tab. 2).

Euploca lagoensis (Warm.) Diane \& Hilger é encontrada desde o México, Antilhas, até o Brasil, estendendo-se nas Regiões Nordeste, CentroOeste e Sudeste. Está associada à vegetação de Caatinga (Piauí, Ceará, Rio Grande do Norte e Paraíba) sobre faixas de dobramento do nordeste oriental, na depressão sertaneja. No estado do
Mato Grosso do Sul ocorre em áreas do Pantanal e em São Paulo no Cerrado, alcançando, neste último estado, o limite meridional de distribuição. Compreende, portanto, uma distribuição geográfica contínua e ampla com o padrão biogeográfico do tipo Cerrado-CaatingaParanaense (Fig. 2, Tab. 2).

Euploca ocellata (Cham.) J.I.M. Melo \& Semir distribui-se na Argentina e, no Brasil, está presente somente no sudoeste do Rio Grande do Sul (Fig. 2). E. krapovickasii J.I.M. Melo \& Semir foi registrada, até o momento, somente no município de Alegrete, associada aos campos alagados. $H$. nicotianaefolium Poir. e $H$. phylicoides Cham., todas encontradas no Rio Grande do Sul, em área que corresponde àregião fisiográfica da Campanha. Os dois primeiros têm distribuição geográfica muito restrita e os dois últimos, distribuição contínua e restrita. Com relação ao padrão biogeográfico aqui adotado e de acordo com Cabrera \& Willink(1980), os quatro táxons estão situados na província Pampeana (Fig. 2, Tab. 2).

Tabela 2- Padrões de distribuição geográfica e biogeográficos das espécies de Euploca Nutt. e Heliotropium L. no Brasil.

Table 2 - Geographic distribution and biogeography patterns of the species of Euploca Nutt. and Heliotropium L. in Brazil.

\begin{tabular}{|c|c|c|}
\hline $\begin{array}{l}\text { Padrão de distribuição } \\
\text { geográfica no Brasil }\end{array}$ & $\begin{array}{l}\text { Padrão biogeográfico } \\
\text { (sensu Cabrera \& Willink 1980) }\end{array}$ & Táxon(s) \\
\hline \multirow[t]{7}{*}{ Contínuo amplo } & $\begin{array}{l}\text { Amazônico-Cerrado-Caatinga-Atlântico- } \\
\text { Paranaense-Pampeano }\end{array}$ & E. procumbens \\
\hline & Amazônico-Cerrado-Atlântico-Paranaense & E. filiformis, $H$. indicum \\
\hline & Amazônico-Atlântico-Caatinga & E. polyphylla \\
\hline & Atlântico-Caatinga-Paranaense & H. angiospermum \\
\hline & Cerrado-Caatinga-Atlântico-Paranaense-Pampeano & E. salicoides \\
\hline & Cerrado-Caatinga-Paranaense & $\begin{array}{l}\text { E. lagoensis, H. elongatum, } \\
\text { H. transalpinum }\end{array}$ \\
\hline & Cerrado & E. parciflora \\
\hline \multirow[t]{4}{*}{ Contínuo restrito } & Caatinga & E. fruticosa \\
\hline & Cerrado-Caatinga & E. paradoxa, E. rodaliae, E. ternata \\
\hline & Pampeano & E. nicotianaefolium, $H$. phylicoides \\
\hline & Pampeano-Paranaense & H. amplexicaule, H. leiocarpum \\
\hline \multirow[t]{3}{*}{ Muito restrito } & Caatinga & E. barbata \\
\hline & Cerrado & $\begin{array}{l}\text { E. hassleriana, E. humistrata, } \\
\text { E. pallescens, E. pottii }\end{array}$ \\
\hline & Pampeano & E. krapovickasii, E. ocellata \\
\hline Disjunto & Caatinga, Pampeano & H. curassavicum \\
\hline
\end{tabular}


Euploca polyphylla (Lehm.) J.I.M. Melo \& Semir se dispersa nas Américas do Norte, Central e do Sul (Johnston 1928) e, no Brasil, ocorre nas Regiões Norte, Nordeste e Sudeste, geralmente associada à Província Atlântica, em vegetação de restinga, especialmente em áreas assentadas nos cordões litorâneos sobre dunas. Esta espécie, ocasionalmente, penetra a oeste, em ambientes de Caatinga. Tal distribuição geográfica, contínua e ampla, constitui o padrão biogeográfico Amazônico-Attântico-Caatinga observado nesta espécie (Fig. 2, Tab. 2).

Euploca procumbens (Mill.) Diane \& Hilger distribui-se desde o sul dos Estados Unidos até Argentina, e nas Antilhas. No Brasil, ocorre nas Regiões Norte, Nordeste, CentroOeste, Sudeste e Sul, o que caracteriza uma distribuição geográfica contínua e ampla e enquadrando-se no padrão biogeográfico Amazônico-Cerrado-Caatinga-AtlânticoParanaense-Pampeano (Fig. 1, Tab. 2).

Euploca ternata (Vahl) J.I.M. Melo \& Semir é encontrada desde o México, Antilhas, Guiana Inglesa e Venezuela, e no Brasil, nas Regiões Nordeste, Centro-Oeste e Sudeste, em ambientes de Caatinga, Cerrado, inclusive em Cerradão e no Pantanal. Isso caracteriza uma distribuição geográfica contínua e ampla, com padrão biogeográfico Cerrado-Caatinga (Fig. 2, Tab. 2).

Heliotropium angiospermum Murray distribui-se desde o sul dos Estados Unidos, alcançando Brasil e Chile (Johnston 1928). No Brasil, ocorre em toda Região Nordeste e no Sudeste nos estados de Minas Gerais e Rio de Janeiro, sendo este último o limite meridional de distribuição da espécie. Está associada à Floresta Atlântica, mas, principalmente, à vegetação de Caatinga hipo-xerofítica e hiperxerofítica, onde é frequentemente encontrada em simpatria com H. elongatum e E. procumbens. Este táxon abrange, portanto, uma distribuição geográfica contínua e ampla, com padrão Atlântico-Caatinga-Paranaense (Fig. 2, Tab. 2).

Dentre as espécies americanas de Heliotropium, H. transalpinum Vell. é a que possui mais ampla distribuição, ocorrendo desde o México, alcançando até a Argentina (Frohlich 1981). No Brasil, está registrada para as Regiões Nordeste, Centro-Oeste, Sudeste e Sul. Este táxon encontra-se associado ao Cerrado, matas mesófilas, Campos Sulinos e, menos frequentemente, em áreas de transição Caatinga-Cerrado no sudoeste baiano. Esta distribuição geográfica contínua e ampla incluise no padrão Cerrado-Caatinga-Paranaense (Fig. 2, Tab. 2).

As espécies Euploca salicoides (Cham.) J.I.M. Melo \& Semir e H. elongatum (Lehm.) I.M. Johnst. distribuem-se, segundo Johnston (1928), na América do Sul, incluindo Bolívia, Paraguai, Argentina, Uruguai e Brasil caracterizando uma distribuição ampla sulamericana e contínua (Fig. 2, Tab. 2). No Brasil ambas apresentando distribuição geográfica contínua e ampla. A primeira ocorre nas Regiões Nordeste, Centro-Oeste, Sudeste e Sul, vinculada à Caatinga, Cerrado, Campos Rupestres, Campos Sulinos ou mais raramente em ambiente de transição Cerrado-Caatinga ou restinga. É a única representante estudada a apresentar o padrão biogeográfico CerradoCaatinga-Atlântico-Paranaense-Pampeano (Fig. 2, Tab. 2). Já a segunda ocorre nas Regiões Norte, Nordeste, Centro-oeste, Sudeste e Sul, associada à Floresta Atlântica, Caatinga, Cerrado e Campos Sulinos e, portanto, engloba o padrão biogeográfico Cerrado-CaatingaParanaense (Fig. 1, Tab. 2) com distribuição contínua e ampla.

Euploca barbata (A. DC.) J.I.M. Melo \& Semir, E. paradoxa (Mart.) J.I.M. Melo \& Semir e E. rodaliae J.I.M. Melo \& Semir são endêmicas do Brasil, estando a primeira associada à Caatinga, enquanto as últimas dispersam-se na Caatinga e Cerrado (Figs. $1 \mathrm{e}$ 2, Tab. 1). Com relação às duas últimas espécies, padrão similar é apresentado pela espécie $E$. ternata, que se distribui nas Regiões Nordeste, Centro-Oeste e Sudeste, em áreas de Caatinga, Cerrado e Pantanal. De acordo com a proposição de Cabrera \& Willink (1980) e Morrone (1999) a ocorrência destes três táxons configura o padrão biogeográfico Cerrado-Caatinga (Fig. 2, Tab. 2), com distribuição contínua e ampla. 
Heliotropium amplexicaule Vahl (Fig. 1, Tab. 1) estende-se desde o sudeste e sul do Brasil, Bolívia, Uruguai até a Argentina (PérezMoreau 1979) e H. leiocarpum (Fig. 2, Tab. 1), distribui-se em toda a Região Sul do Brasil, Paraguai e Argentina. De acordo com o proposto por Cabrera \& Willink (1980) e Morrone (1999), ambos os táxons se caracterizam pelo padrão Pampeano-Paranaense (Figs. $1 \mathrm{e}$ 2, Tab. 2) com distribuição geográfica contínua e restrita. $\mathrm{O}$ padrão muito restrito também denominado de "endemismo local" (Gentry 1982) ou "micro-endemismo" (Pirani 1990) engloba cinco espécies: E. hassleriana (Chodat) J.I.M. Melo \& Semir, E. humistrata (Cham.) J.I.M. Melo \& Semir, E. krapovickasii J.I.M. Melo \& Semir, E. pallescens (I.M. Johnst.) J.I.M. Melo \& Semir e E. pottii J.I.M. Melo \& Semir. Euploca hassleriana, E. pallescens e E. pottii foram encontradas apenas no estado do Mato Grosso do Sul, sendo a primeira associada à vegetação de Cerrado no sudoeste do estado, enquanto as demais estão relacionadas às planícies do Pantanal (Fig. 1). Este conjunto de espécies enquadrase no padrão biogeográfico Cerrado sensu Cabrera \& Willink (1980). De acordo com esses mesmos autores, E. humistrata se caracteriza pelo padrão Cerrado (Fig. 1, Tab. 2), enquanto E. krapovickasii abrange o padrão Pampeano (Fig. 2, Tab. 2).

As espécies de Euploca e Heliotropium são na maioria neotropicais, com exceção apenas $H$. curassavicum e $H$. indicum, que estão amplamente distribuídas nas regiões tropicais do globo. Heliotropium curassavicum é uma espécie exclusivamente halófila enquanto $H$. indicum é geralmente encontrada como ruderal (Miller 1988), associada, no Brasil, à borda de florestas mesófilas ou de terras baixas (Melo \& Semir 2008).

No Brasil, E. filiformis, E. lagoensis, E. polyphylla, E. procumbens, E. salicoides e $E$. ternata são as espécies que apresentaram maior área de ocorrência. Euploca procumbens está associada a todos os tipos vegetacionais. Euploca filiformis, E. procumbens e E. ternata são amplamente distribuídas em toda a América tropical, apresentando áreas de ocorrência quase coincidentes ao longo da sua distribuição.

Os estados da Bahia e Rio Grande do Sul reúnem o maior número de representantes dos gêneros estudados, com 13 e 11 espécies, respectivamente, seguidos pelos estados de Pernambuco e Minas Gerais, com dez espécies cada um.

Com relação às regiões geopolíticas, as espécies estudadas encontram-se assim distribuídas: Norte, com sete espécies (Euploca (5) e Heliotropium (2)); Nordeste, com 16 espécies (Euploca (11) e Heliotropium (5)); Centro-Oeste, com 15 espécies (Euploca (11) e Heliotropium (4)); Sudeste, representada por 11 espécies (Euploca (6) e Heliotropium (5)) e Sul, com 12 espécies (Euploca (4) e Heliotropium (8)). Euploca procumbens, $H$. elongatum e $H$. indicum são as únicas espécies encontradas em todas as Regiões do Brasil. A maioria das espécies de Heliotropium (oito), no entanto, ocorre na Região Sul, exceção para $H$. angiospermum com o limite meridional de distribuição no estado do Rio de Janeiro.

A maioria das espécies estudadas (15) ocorre na Caatinga, dez das quais pertencentes à Euploca e cinco a Heliotropium, das quais E. barbata e E. fruticosa são exclusivas a este bioma. A Província Cerrado engloba 12 espécies, nove delas pertencentes à Euploca e três a Heliotropium. No que se referem às espécies representadas no Pantanal, seis pertencem a Euploca e duas a Heliotropium, sendo Euploca pallescens e E. pottii restritas ao bioma. Esta última, até o momento, é considerada como endêmica do Brasil.

A Floresta Amazônica engloba a menor riqueza de espécies (quatro) para os gêneros estudados; três delas pertencentes a Euploca (E. filiformis, E. polyphylla e E. procumbens), e uma espécie cosmopolita de Heliotropium (H. indicum). Estas espécies, dentre as estudadas, compreendem as de maior área de ocorrência. A inexpressiva diversidade de espécies de Euploca e Heliotropium na Floresta Amazônica provavelmente reside no fato de se tratar de área constituída por ambientes em sua maioria, florestais. 
Na Região Sul do Brasil, foram encontradas 11 espécies, o maior número (oito) observado em Heliotropium. Os Campos Sulinos compreendem também o centro de diversidade das espécies de Heliotropium sect. Heliotrophytum G. Don, todas elas representadas nessa região $(H$. amplexicaule, $H$. leiocarpum, $H$. nicotianeafolium e $H$. phylicoides), inclusive no Rio Grande do Sul. Ainda no que se refere às espécies da secção Heliotrophytum, os padrões biogeográficos aqui detectados nas mesmas foram também observados em Crotalaria incana L. - Leguminosae (Flores \& Miotto 2005) e Aristida flaccida Trin. \& Rupr. - Poaceae (Longhi-Wagner 1990), ambas distribuídas em toda a região Sul e $A$. teretifolia Arech. encontrada apenas no Rio Grande do Sul (Longhi-Wagner 1990). Este aspecto faz supor que o estabelecimento dessas espécies, na Região Sul, esteja provavelmente associado ao clima temperado superúmido.

Heliotropium amplexicaule e $H$. leiocarpum são as únicas espécies a apresentarem padrão biogeográfico Paranaense-Pampeano, similar ao registrado por Loiola (2001), para Erythroxylum myrsinites Mart. e por Sakuragui (2001), para Philodendron appendiculatum Nadruz \& Mayo e P. loefgrenii Engler-Araceae.

Quatro espécies caracterizam-se pelo padrão de distribuição biogeográfico Pampeano: $E$. krapovickasii, E. ocellata, H. phylicoides e $H$. nicotianaefolium, também referido para espécies de Eragrostis e Stipa - Poaceae (Boechat \& Longhi-Wagner 2000; LonghiWagner \& Zanin 1998).

O padrão Atlântico-Caatinga registrado para Euploca polyphylla e Heliotropium angiospermum foi também mencionado para representantes de diversas famílias, como Apocynaceae, Bignoniaceae, Caesalpiniaceae, Capparaceae, Euphorbiaceae, Fabaceae, Malvaceae, Myrtaceae, Orchidaceae e Rubiaceae (Rodal et al. 2005).

O padrão Cerrado-Caatinga-Paranaense detectado, neste estudo, em E. lagoensis, $H$. elongatum e $H$. transalpinum foi também referido para Froelichia procera (Seub.) Pedersen - Amaranthaceae por Marchioretto et al. (2004).
O padrão Cerrado-Caatinga corresponde, no Brasil, à área de ocupação dos biomas do Cerrado e da Caatinga e, neste estudo, inclui três espécies: Euploca paradoxa, E. rodaliae e E. ternata. Dentre elas, esta última é a que apresenta maior área de ocorrência, estendendose desde o México até o Brasil, enquanto as duas primeiras são endêmicas do Brasil. Euploca paradoxa está associada, especialmente às coberturas metassedimentares das bacias do rio São Francisco, no nordeste do Brasil ou, também, ao Planalto Central na Região CentroOeste. Euploca rodaliae configura a vegetação de Caatinga nos estados de Pernambuco e Bahia ou, mais frequentemente, em áreas de Cerrado, no estado da Bahia onde alcança seu limite meridional de distribuição. Este mesmo padrão de distribuição foi encontrado por Silva \& Sales (2008) para Phyllanthus claussenii Müll. Arg. - Phyllanthaceae, e também por Marchioretto et al. (2004) em Froelichia humboldtiana (Roem. \& Schult.) Seub. - Amaranthaceae, espécie encontrada nas Regiões Nordeste e Sudeste do Brasil.

Euploca hassleriana, E. humistrata, E. pallescens, E. parciflora e E. pottii apresentaram o padrão de distribuição biogeográfico do tipo Cerrado, similar ao encontrado por Cordeiro (1990) para Julocroton humilis Diedr. - Euphorbiaceae, Giulietti \& Pirani (1988) paraespécies pertencentes a distintas famílias, e Wanderley (1990) para Orthophytum mello-barretoi L.B. Sm. Bromeliaceae. Dentre essas espécies, $E$. parciflora é a única espécie a apresentar distribuição contínua e ampla, enquanto as demais possuem distribuição muito restrita (endemismo local ou regional) (Fig. 1, Tab. 2).

No padrão Caatinga, foram enquadradas apenas duas espécies: E. barbata e E. fruticosa (Fig. 2, Tab. 2), sendo este mesmo padrão biogeográfico detectado para outras espécies de Boraginaceae s.l. por Prado \& Gibbs (1993), dentre as quais se sobressaem Cordia dardani Taroda, Auxemma oncocalyx (Allemão) Taub. e Patagonula bahiensis Moric., exclusivas ao domínio das Caatingas, e por Loiola (2001), para diversas espécies de Erythroxylum Erythroxylaceae. 
Dentre as espécies que apresentam padrão tropical, destaca-se no gênero Euploca, um grupo constituído por plantas exclusivamente herbáceas, frequentemente prostradas, com flores solitárias, longamente pediceladas, axilares ou supra-axilares. Distribuem-se nas Américas do Norte (México), Central (Panamá e Guatemala, incluindo Antilhas) e Sul (Brasil, Bolívia, Colômbia e Venezuela), África Tropical, Madagascar e Índia e, provavelmente, na Austrália. Reúne aproximadamente oito espécies, quatro das quais ocorrentes no Brasil: E. lagoensis, E. humistrata, E. paradoxa e E. parciflora, sendo as três últimas endêmicas (Melo \& Semir com. pess.). Euploca lagoensis é a que apresenta maior área de ocorrência, estendendo-se desde o México, incluindo Antilhas, até o Brasil.

As espécies dos gêneros Euploca e Heliotropium se estabelecem predominantemente em ambientes abertos nas zonas áridas e semiáridas do globo. Tais preferências foram também constatadas no Brasil, verificando-se que no gênero Euploca as espécies ocorrem geralmente nas Regiões Nordeste e Brasil Central, em formações abertas (Caatinga e Cerrado). Com relação à Heliotropium, suas espécies encontramse associadas, principalmente, ao Planalto Meridional em ambientes abertos, secos ou, mais raramente, em campos alagadiços. Ambos os gêneros concentram táxons algumas vezes restritos a essas formações. As condições ambientes de ocorrência das espécies aqui tratadas assim como os fatores abióticos relacionados, quando conhecidos, foram citadas por Melo \& Semir (2008) e Melo \& Semir (no prelo).

Considerando-se que, no Brasil, o estabelecimento das espécies de Euploca e Heliotropium dá-se geralmente em ambientes xéricos, como também o amplo espectro de distribuição e habitat da maioria delas, é interessante mencionar que as mesmas apresentam-se fortemente vinculadas a cursos de água. Este aspecto sugere que a água provavelmente representa um dos fatores determinantes para o estabelecimento de suas espécies, ainda mais se considerarmos a morfologia carpológica das mesmas. Os frutos são esquizocárpicos o que favorece a dispersão dos diásporos a longa distância por hidrocoria e também através de endozoocoria (pássaros e mamíferos). A hidrocoria foi mencionada em H. curassavicum (Lesko \& Walker 1969), $H$. indicum e $H$. nicotianaefolium, todas aqui estudadas. Essa síndrome de dispersão também ocorre em espécies de Euploca, haja vista a presença de câmaras aeríferas nos frutos de algumas delas, que promove o transporte dos frutos ou das núculas pela água. Tal aspecto se torna evidente, sobretudo em E. paradoxa, que habita as margens do rio São Francisco no estado da Bahia. Euploca filiformis, E. lagoensis e E. procumbens também se apresentam geralmente associadas a áreas com grande disponibilidade hídrica, especialmente no complexo pantaneiro ou, menos frequentemente, na Floresta Amazônica.

Também no que se refere à dispersão, um outro aspecto importante desta vez observado, nos trópicos, por Snow (1981) é a ingestão das núculas de espécies de Heliotropium por pássaros frugívoros não especializados. Este mecanismo também foi verificado por Barker \& Vestjens $(1989,1990)$ apud Craven (1996) em representantes australianos de Heliotropium e, sugerido por Böhle et al. (1996), em espécies insulares do gênero Echium L. (Boraginaceae s.s.). Outros exemplos de ornitocoria foram ainda observados em Cordiaceae, nas espécies Cordia multispicata Cham. e C. nodosa Lam., em uma área de Floresta Atlântica em Pernambuco (Griz \& Machado 1998), e por Gottschling et al. (2004), que também mencionaram a endozoocoria por mamíferos, para explicar a dispersão à longa distância em Heliotropiaceae. A mirmecocoria é referida por Craven (1996) para espécies australianas de Heliotropium s.l. (incluindo Euploca). Esse mecanismo deve estar relacionado à presença de cavidades na região dorsal das núculas, observadas na maioria das espécies de Euploca (Heliotropium sect. Orthostachys R.Br.), o que promove, portanto, o transporte desses diásporos por formigas. Os mecanismos supramencionados corroboram o amplo espectro de distribuição geográfica observada em algumas das espécies estudadas. 


\section{Agradecimentos}

À CAPES (Coordenação de Aperfeiçoamento de Pessoal de Nível Superior) a concessão da bolsa de doutoramento ao primeiro autor. Ao Programa de Pós-Graduação em Botânica da Universidade Federal Rural de Pernambuco, Recife, PE, Brasil as facilidades concedidas durante a realização deste trabalho. Aos curadores dos herbários nacionais e estrangeiros o envio das coleções através de empréstimo e ou doação ou a permissão para consulta das mesmas.

\section{REFERÊNCIAS BIBLIOGRÁFICAS}

Acevedo-Rodríguez, P. 1990. Distributional patterns in Brazilian Serjania (Sapindaceae). Acta Botanica Brasilica 4(1): 69-82.

Akhani, H. 2007. Diversity, biogeography, and photosynthetic pathways of Argusia and Heliotropium (Boraginaceae) in South-West Asia with an analysis of phytogeographical units. Botanical Journal of the Linnean Society 155: 401-425.

Barros, F. 1990. Diversidade taxonômica e distribuição geográfica das Orchidaceae brasileiras. Acta Botanica Brasilica 4(1): 177-187.

Boechat, S.C. \& Longhi-Wagner, H.M. 2000. Padrões de distribuição geográfica dos táxons brasileiros de Eragrostis (Poaceae, Chloridoideae). Revista Brasileira de Botânica 23(2): 177-194.

Böhle, U.-R.; Hilger, H.H. \& Martin, W.F. 1996. Island colonization and evolution of the insular woody habit in Echium L. (Boraginaceae). Proceedings of the National Academy Sciences of the United States of America 93(21): 11740-11745.

Cabrera, A.L. \& Willink, A. 1980. Biogeografia de América Latina. Secretaria Geral de la Organización de los Estados Americanos, Washington. 117p.

Cordeiro, I. 1990. Aspectos taxonômicos e distribuição geográfica de Julocroton Mart. (Euphorbiaceae). Acta Botanica Brasilica 4(1): 245-249.

Craven, L.A. 1996. A taxonomic revision of Heliotropium (Boraginaceae) in Australia. Australian Systematic Botany 9: 521-657.

Flores, A.S. \& Miotto, S.T.S. 2005. Aspectos fitogeográficos das espécies de Crotalaria $\mathrm{L}$. (Leguminosae, Faboideae) na Região Sul do Brasil. Acta Botanica Brasilica 19(2): 245-249.
Frohlich, M.W. 1981. Heliotropium. In: Nash, D. L. \& Moreno, N. P. (eds.). Flora de Veracruz: Boraginaceae. Instituto Nacional de Investigaciones sobre Recursos Bióticos, Xalapa v. 18. Pp. 70-104.

Gentry, A. 1982. Phytogeographical patterns in Northwestern South America and Southern Central America as evidence for a Chocó Refugium. In: Prance, G. (ed.). Biological diversification in the tropics. Columbia University Press, New York. Pp. 112-136.

Giulietti, A.M. \& Pirani, J.R. 1988. Patterns of geographic distribution of some plant species from the Espinhaço range, Minas Gerais and Bahia, Brazil. In: Heyer, W.R. \& Vanzolini, P.E. (eds.). Proceedings of a workshop on Neotropical distribution patterns. Academia Brasileira de Ciências, Rio de Janeiro. Pp. 39-69.

Giulietti, A.M.; Harley, R.M.; Queiroz, L.P.; Wanderley, M.G.L. \& van den Berg, C. 2005. Biodiversidade e conservação das plantas no Brasil. In: Silva, J.M.C.; Brandon, K.; Fonseca, G. \& Rylands, A. (eds.). Desafios e oportunidades para a conservação da Biodiversidade no Brasil. Megadiversidade 1: 52-61.

Gottschling, M.; Diane, N.; Hilger, H.H. \& Weigend, M. 2004. Testing hypotheses on disjunctions present in the primarily woody Boraginales: Ehretiaceae, Cordiaceae, and Heliotropiaceae, inferred from ITSI sequence data. International Journal of Plant Science 165: S123-S135.

Griz, L.M.S. \& Machado, I.C.S. 1998. Aspectos morfológicos e síndromes de dispersão de frutos e sementes na Reserva Ecológica de Dois Irmãos. In: Machado, I. C. S.; Lopes, A. V. \& Porto, K. C. (eds.). Reserva Ecológica de Dois Irmãos: Estudos em um remanescente de Mata Atlântica em área urbana (Recife-PernambucoBrasil). Editora Universitária da Universidade Federal de Pernambuco, Recife. Pp.197-224.

Hilger, H.H. \& Diane, N. 2003. A systematics analysis of Heliotropiaceae (Boraginales) based on $\operatorname{trn} \mathrm{L}$ and ITSI sequence data. Botanische Jahrbürcher für Pflanzengeschichte und Pflanzengeographie 125: $19-51$.

Holmgren, P.K.; Holmgren, N.H. \& Barnett, L.C. (eds.). 2006. Index Herbariorum. <http://sciweb. nybg.org./science2/IndexHerbariorum.asp>. Acessado em 08 março 2006.

Johnston, I.M. 1928. Studies in Boraginaceae VII. The South American species of Heliotropium. 
Contributions from the Gray Herbarium of Harvard University 81: 3-83.

Lesko, G.L. \& Walker, R.B. 1969. Effects of sea water on seed germination in two Pacific atoll beach species. Ecology 50: 730-734.

Loiola, M.I. B. 2001. Revisão taxonômica de Erythroxylum P. Browne sect. Rhabdophyllum O.E. Schulz (Erythroxylaceae Kunth). Tese de Doutorado. Universidade Federal Rural de Pernambuco, Recife. 238p.

Longhi-Wagner, H.M. 1990. Diversidade e distribuição geográfica das espécies de Aristida L. (Gramineae) ocorrentes no Brasil. Acta Botanica Brasilica 4(1): 105-124.

Longhi-Wagner, H.M. \& Zanin, A. 1998. Padrões de distribuição geográfica das espécies de Stipa L. (Poaceae-Stipeae) ocorrentes no Brasil. Revista Brasileira de Botânica 21: 167-175.

Marchioretto, M.S.; Windisch, P.G. \& Siqueira, J.C. 2004. Padrões de distribuição geográfica de Froelichia Moench e Froelichiella R.E. Fries (Amaranthaceae) no Brasil. Iheringia, Ser. Botânica, 59(2): 149-159.

Melo, J.I.M. \& Andrade, W.M. 2007. Boraginaceae s.l. A. Juss. em uma área de Caatinga da ESEC Raso da Catarina, BA, Brasil. Acta Botanica Brasilica 21(2): 369-378.

Melo, J.I.M. \& Sales, M.F. 2005. Boraginaceae A. Juss. na região de Xingó: Alagoas e Sergipe. Hoehnea 32(3): 369-380.

Melo, J.I.M. \& Semir, J. 2008. Taxonomia do gênero Heliotropium L. (Heliotropiaceae) no Brasil. Acta Botanica Brasilica 22(3): 754-770.

Melo, J.I.M. \& Semir, J. 2010. Taxonomia do gênero Euploca Nutt. (Heliotropiaceae) no Brasil. Acta Botanica Brasilica 24(1): no prelo.

Miller, J.S. 1988. A revised treatment of Boraginaceae for Panama. Annals of the Missouri Botanical Garden 75: 456-521.

Miller, J.S. 2003. A new species of Heliotropium L. (Boraginaceae) from Madagascar. Adansonia 25: $115-118$.

Morrone, J. 1999. Presentación preliminar de un nuevo esquema biogeográfico de America del Sur. Comptes Rendus Sommaires des Séances de la Société de Biogeographie 75(1): 1-16.

Pérez-Moreau, R.L. 1979. Boraginaceae. In: Burkart, A. (ed.). Flora ilustrada de Entre Rios, Argentina.
Instituto Nacional de Tecnologia Agropecuária, Buenos Aires, v. 6, n. 5. Pp. 209-229.

Pirani, J.R. 1990. Diversidade taxonômica e padrões de distribuição geográfica em Picramnia (Simaroubaceae) no Brasil. Acta Botanica Brasilica 4(1): 19-44.

Prado, D. \& Gibbs, P.E. 1993. Patterns of species distributions in the dry seasonal forests of South America. Annals of the Missouri Botanical Garden 80: 902-927.

Ridley, H.N. 1930. The dispersal of plants throughout the world. L. Reeve \& Co, Ashford. 744p.

Rodal, M.J.N.; Sales, M.F.; Silva, M.J. \& Silva, A.G. 2005. Flora de um Brejo de Altitude na escarpa oriental do planalto da Borborema, PE, Brasil. Acta Botanica Brasilica 19(4): 843-858.

Sakuragui, C.M. 2001. Biogeografia de Philodendron seção Calostigma (Schott) Pfeiffer (Araceae) no Brasil. Acta Scientiarum, Ser. Ciências Biológicas, 23(2): 561-569.

Silva, M.J. \& Sales, M.F. 2008. Sinopse do gênero Phyllanthus L. (Phyllanthaceae) no Nordeste do Brasil. Rodriguésia 59(2): 407-422.

Snow, D.W. 1981. Tropical frugivorous birds and their food plants: a world survey. Biotropica 13(1): 1-14.

Sylvestre, L.S. 2002. Estudos taxonômicos e florísticos das pteridófitas brasileiras: desafios e conquistas. In: Araújo, E.L.; Moura, A.N.; Sampaio, E.V.S.B.; Gestinari, L.M.S. \& Carneiro, J.M.T. (eds.). Biodiversidade, conservação e uso sustentável da flora do Brasil. LIII Congresso Nacional de Botânica/XXV Reunião Nordestina de Botânica. Recife. Pp.194-195.

Thomas, W.W. \& Magill, R.E. 2002. Uma proposta para um check-list on line das plantas neotropicais. In: Araújo, E.L.; Moura, A.N.; Sampaio, E.V.S.B.; Gestinari, L.M.S. \& Carneiro, J.M.T. (eds.). Biodiversidade, conservação e uso sustentável da flora do Brasil. LIII Congresso Nacional de Botânica/XXV Reunião Nordestina de Botânica. Recife. Pp.252-254.

Wanderley, M.G.L. 1990. Diversidade e distribuição geográfica das espécies de Ortophytum (Bromeliaceae). Acta Botanica Brasilica 4(1): 169-175. 